\title{
Fraturas do fêmur em idosos do Nordeste do Brasil: dados epidemiológicos e gastos
}

\section{para o SUS}

\author{
Femur fractures in the elderly in Northeast Brazil: epidemiological data and expenses for the SUS \\ Fracturas del fémur en ancianos del Noreste de Brasil: datos epidemiológicos y gastos del SUS
}

Recebido: 18/10/2021 | Revisado: 25/10/2021 | Aceito: 26/10/2021 | Publicado: 30/10/2021

\author{
José Ernando dos Santos Júnior \\ ORCID: https://orcid.org/0000-0002-6250-4250 \\ Universidade Tiradentes, Brasil \\ E-mail: jose.ernando@ souunit.com.br \\ Ronald Bispo Barreto da Silva \\ ORCID: https://orcid.org/0000-0002-8500-1904 \\ Universidade Tiradentes, Brasil \\ E-mail: dr.ronaldbarreto@gmail.com
}

\begin{abstract}
Resumo
Objetivos: avaliar dados epidemiológicos incluindo incidência, faixa etária, sexo, cor/raça, mortalidade, média de permanência em internação e os gastos para o Sistema Único de Saúde (SUS) relativos às fraturas de fêmur em idosos ( $\geq 60$ anos) do Nordeste do Brasil. Métodos: estudo descritivo feito a partir de dados secundários provenientes do Sistema de Informações Hospitalares do Sistema Único de Saúde (SIH/SUS). Na plataforma DATASUS foram selecionados filtros representando as variáveis do estudo relacionadas ao período compreendido entre janeiro de 2019 a dezembro de 2020. Os cálculos de incidência foram feitos com base na população da região Nordeste definida pelo censo de 2010 do IBGE. Resultados: houveram 24239 internações por fratura de fêmur no intervalo de tempo estudado. A incidência anual média foi de 22,22 casos a cada 10000 idosos nordestinos. Esses números geraram um custo total ao SUS de R\$ 62.713.218,03. Idosos com idade igual ou superior a 80 anos foram a maioria, protagonizando $48,17 \%$ das internações. As mulheres apresentaram 2,2 vezes mais internações que os homens. Os homens apresentaram uma taxa de mortalidade maior, 3,97\%. Pardos totalizaram a maior parte das internações. Indígenas e brancos apresentaram maior mortalidade. Conclusão: as fraturas de fêmur apresentam elevada incidência em idosos do Nordeste do Brasil e geram gastos onerosos para o sistema de saúde.
\end{abstract}

Palavras-chave: Fraturas do fêmur; Osteoporose; Idoso; Incidência; Gastos em saúde.

\begin{abstract}
Objectives: to evaluate epidemiological data including incidence, age group, sex, color / race, mortality, average hospital stay and expenses of the Unified Health System (SUS) related to femur fractures in the elderly ( $\geq 60$ years) in the Northeast of Brazil. Methods: descriptive study based on secondary data from the Hospital Information System of the Unified Health System (SIH / SUS). In the DATASUS platform, filters were selected that represent the study variables related to the period from January 2019 to December 2020. The incidence calculations were made based on the population of the Northeast region defined by the 2010 IBGE census. Results: there were 24,239 hospitalizations for femur fractures during the time period studied. The mean annual incidence was 22.22 cases per 10,000 elderly people in the Northeast. These figures generated a total cost for the SUS of R \$62,713,218.03. People aged 80 years and over were the majority, with $48.17 \%$ of income. Women had 2.2 times more hospitalizations than men. Men had a higher mortality rate, $3.97 \%$. Browns accounted for the majority of admissions. Indigenous and whites had higher mortality. Conclusion: femur fractures have a high incidence in the elderly in northeastern Brazil and generate costly expenses for the health system.
\end{abstract}

Keywords: Femoral fractures; Osteoporosis; Elderly; Incidence; Health expenditures.

\section{Resumen}

Metas: evaluar datos epidemiológicos incluyendo incidencia, grupo de edad, sexo, color / raza, mortalidad, estadía hospitalaria promedio y gastos del Sistema Único de Salud (SUS) relacionados con fracturas de fémur en ancianos ( $\geq 60$ años) en el Nordeste de Brasil. Métodos: estudio descriptivo basado en datos secundarios del Sistema de Información Hospitalaria del Sistema Único de Salud (SIH / SUS). En la plataforma DATASUS se seleccionaron filtros que representan las variables de estudio relacionadas con el período de enero de 2019 a diciembre de 2020 . Los cálculos de incidencia se realizaron con base en la población de la región Nordeste definida por el censo del IBGE 2010. Resultados: hubo 24239 hospitalizaciones por fracturas de fémur durante el período de tiempo estudiado. La incidencia media anual fue de 22,22 casos por cada 10.000 ancianos del Nordeste. Estas cifras generaron un costo total para el SUS de R\$ 62.713.218,03. Las personas mayores de 80 años y más fueron la mayoría, con el 48,17\% de 
los ingresos. Las mujeres tuvieron 2,2 veces más hospitalizaciones que los hombres. Los hombres tenían una tasa de mortalidad más alta, 3,97\%. Los marrones representaron la mayoría de las admisiones. Los indígenas y los blancos tenían una mayor mortalidad. Conclusión: las fracturas de fémur tienen una alta incidencia en los ancianos del noreste de Brasil y generan costosos gastos para el sistema de salud.

Palabras clave: Fracturas del fémur; Osteoporosis; Anciano; Incidencia; Gastos en salud.

\section{Introdução}

A população idosa cresce significativamente no Brasil, sendo o conceito de idoso adotado no país o da Organização Mundial da Saúde: o indivíduo de 60 anos de idade ou mais, se ele residir em países em desenvolvimento (Miranda et al., 2016). Segundo projeções do Instituto Brasileiro de Geografia e Estatística em 2060, cerca de um quarto da população brasileira será composta por idosos (IBGE, [s.d.]). O crescimento da população idosa gera uma série de alterações em variados âmbitos da sociedade como na economia, no mercado de trabalho e nos sistemas e serviços de saúde (Miranda et al., 2016).

Dos impactos do envelhecimento nos serviços de saúde, merece destaque a osteoporose. A osteoporose é considerada uma doença decorrente de múltiplos mecanismos patogenéticos que resultam em perda de massa óssea e na deterioração da microarquitetura da estrutura esquelética, com consequente aumento da propensão para fraturas de fragilidade (Kanis et al., 2019). É uma doença mais prevalente em mulheres e tende a aumentar com a idade, demonstrando um aumento de $4 \% \mathrm{em}$ mulheres de 50 a 59 anos para 52\% em mulheres de 80 anos ou mais (Barrionuevo et al., 2019).

De acordo com o estudo BRAZOS, os principais locais das fraturas por baixo impacto são o antebraço distal, fêmur, úmero, costelas e vértebra; a fratura de fêmur foi a segunda mais comum, atrás apenas da do antebraço distal (Pinheiro et al., 2010). Uma revisão recente relatou que as fraturas de quadril (fêmur proximal) ocorreram em $28 \%$ das mulheres com osteoporose (Barrionuevo et al., 2019).

As fraturas do fêmur têm grande impacto social e geram despesas onerosas para o sistema de saúde devido a procedimentos cirúrgicos extensos, longos períodos de hospitalização e recuperação do paciente e ao potencial de complicações, sequelas e mortes inerentes a essa condição (Vasconcelos et al., 2020).

As taxas médias de mortalidade em uma revisão publicada em 2004, foram de 5,5\% durante a internação hospitalar, $4,7 \%$ ao fim de um mês de seguimento, $11,9 \%$ com três meses, $10,8 \%$ com seis meses, $19,2 \%$ com um ano e $24,9 \%$ com dois anos, mostrando o potencial que este problema de saúde tem de complicar a curto e longo prazo (Sakaki et al., 2004). O custo médio anual do SUS, entre os anos de 2008 e 2018, para o tratamento das fraturas de fêmur em idosos foi de R\$ 99.718.574,30; o índice de mortalidade hospitalar no mesmo estudo foi de 4,99\%, para 478.274 mil internações registradas no período (Vasconcelos et al., 2020).

Tendo em vista a dimensão do problema que são as fraturas de fêmur em idosos para o sistema de saúde, este estudo busca trazer dados epidemiológicos mais recentes sobre o tema, nos anos de 2019 e 2020, porém restritos à região Nordeste do Brasil.

\section{Metodologia}

Trata-se de um estudo descritivo (Lima-Costa \& Barreto, 2003) feito a partir de dados secundários provenientes do Sistema de Informações Hospitalares do Sistema Único de Saúde (SIH/SUS).

Os dados foram coletados do Departamento de Informática do Sistema Único de Saúde do Brasil (DATASUS) em 05 de setembro de 2021, acessando na seguinte sequência: TABNET; Epidemiológicas e Morbidade; Morbidade Hospitalar do SUS (SIH/SUS); Geral, por local de residência - a partir de 2008; Brasil por Região e Unidade de Federação. Foram selecionados os filtros de "Conteúdo" que representam as variáveis do estudo: Internações; Valor total; Valor médio internação; Média permanência; Óbitos e Taxa mortalidade. O período escolhido foi o de janeiro de 2019 a dezembro de 2020 
e a região apenas a Nordeste. O diagnóstico selecionado, fratura do fêmur, com base no CID-10 (10 revisão da Classificação Estatística Internacional de Doenças e Problemas Relacionados à Saúde): 72.0 a 72.9. Foram feitas as buscas por faixa etária a partir de 60 anos (60 a 69; 70 a 79 e 80 anos ou mais), por sexo e por cor/raça.

Os cálculos de incidência foram realizados considerando a população de 60 anos ou mais do Nordeste do Brasil de acordo com o censo de 2010 do Instituto Brasileiro de Geografia e Estatística (IBGE). O Microsoft Excel ${ }^{\circledR} 2016$ foi utilizado para agrupar os dados em tabelas e para auxiliar nos cálculos.

Não foi necessária a aprovação do Comitê de Ética e Pesquisa porque foram utilizados dados secundários de uma plataforma de domínio público.

\section{Resultados}

De Janeiro de 2019 a Dezembro de 2020, foram registradas 24239 internações nos hospitais do SUS por fratura de fêmur em idosos (60 anos ou mais) residentes na região Nordeste do Brasil. A quantidade de casos em cada ano foi bastante similar, resultando em uma incidência anual média aproximada de 22,22 casos a cada 10000 idosos nordestinos. A média de permanência desses individuos nos hospitais diminuiu aproximadamente um dia em 2020, comparado a 2019. Apesar da quantidade de casos menor em 2020, a mortalidade foi ligeiramente maior. Esses números registrados geraram custos totais de $\mathrm{R} \$ 62.713 .218,03$ ao SUS, originando uma média de $\mathrm{R} \$ 2587,29$ por idoso internado. (Tabela 1)

Tabela 1 - Internações hospitalares devido a fratura de fêmur de idosos residentes na região nordeste, 2019 E 2020

\begin{tabular}{llllllll}
\hline Ano & $\begin{array}{l}\text { Número de } \\
\text { internações }\end{array}$ & $\begin{array}{l}\text { População } \\
\text { de 60 anos } \\
\text { ou mais do } \\
\text { Nordeste } \\
\text { (censo 2010 }\end{array}$ & $\begin{array}{l}\text { Incidência x } \\
\mathbf{1 0 0 0 0}\end{array}$ & $\begin{array}{l}\text { Média de } \\
\text { permanência } \\
\text { (dias) }\end{array}$ & Óbitos & $\begin{array}{l}\text { Taxa de } \\
\text { mortalidade }\end{array}$ & $\begin{array}{l}\text { Custos (reais - } \\
\text { R\$) }\end{array}$ \\
\hline $\mathbf{2 0 1 9}$ & 12208 & 5.452 .124 & 22,39 & 9,3 & 451 & 3,69 & $31.520 .444,83$ \\
$\mathbf{2 0 2 0}$ & 12031 & 5.452 .124 & 22,06 & 8,6 & 468 & 3,89 & $31.192 .773,20$ \\
Total & 24239 & - & - & 8,9 & 919 & 3,79 & $62.713 .218,03$ \\
\hline
\end{tabular}

Fonte: https://datasus.saude.gov.br.

Os números de internações, dias de permanência e de óbitos aumentaram de acordo com a faixa etária, sendo maiores nos idosos com idade igual ou superior a 80 anos, que protagonizaram $48,17 \%$ desses casos de fratura. (Tabela 2 )

Tabela 2 - Internações hospitalares de acordo com a faixa etária.

\begin{tabular}{lllll}
\hline Faixa etária & $\begin{array}{l}\text { Número de internações } \\
\text { (valor absoluto e \%) }\end{array}$ & $\begin{array}{l}\text { Média de } \\
\text { permanência (dias) }\end{array}$ & Óbitos & Taxa de Mortalidade \\
\hline $\mathbf{6 0 - 6 9}$ anos & $4807(19,84 \%)$ & 8,6 & 74 & 1,54 \\
$\mathbf{7 0 - 7 9}$ anos & $7755(31,99 \%)$ & 8,9 & 174 & 2,24 \\
$\geq \mathbf{8 0}$ anos & $11.677(48,17 \%)$ & 9,1 & 671 & 5,75 \\
Total $(\geq \mathbf{6 0}$ anos) & $24239(100 \%)$ & 8,9 & 919 & 3,79 \\
\hline
\end{tabular}

Fonte: https://datasus.saude.gov.br 
Durante esses dois anos foram observadas mais internações em mulheres, 16729 casos, cerca de 2,2 vezes mais internações se comparado aos homens. A faixa etária maior ou igual a 80 anos se mostrou a mais prevalente com 8738 casos. Apesar de as mulheres apresentarem valores absolutos maiores de óbitos em todas as faixas etárias, os homens apresentaram taxas de mortalidade maiores. (Tabela 3)

Tabela 3 - Internações hospitalares de acordo com sexo e idade.

\begin{tabular}{llll}
\hline Sexo & Internações & Óbitos & Taxa de mortalidade \\
\hline Masculino & & & 1,56 \\
$\mathbf{6 0 - 6 9}$ anos & 2181 & 34 & 2,47 \\
$\mathbf{7 0 - 7 9}$ anos & 2390 & 59 & 6,98 \\
$\geq \mathbf{8 0}$ anos & 2939 & 205 & 3,97 \\
Total $(\geq \mathbf{6 0}$ anos) & 7510 & 298 & 1,52 \\
\hline Feminino & & & 2,14 \\
$\mathbf{6 0 - 6 9}$ anos & 2.626 & 40 & 5,33 \\
$\mathbf{7 0 - 7 9}$ anos & 5.365 & 115 & 3,71 \\
$\mathbf{Z} \mathbf{8 0}$ anos & 8738 & 466 & 621 \\
Total $(\geq \mathbf{6 0}$ anos) & 16729 & 621 & \\
\hline
\end{tabular}

Fonte: https://datasus.saude.gov.br

Os indivíduos considerados pardos totalizaram a maior parte das internações e do número absoluto de óbitos, 14081 e 511, respectivamente. Contudo, a maior taxa de mortalidade encontrada foi a de indígenas, provavelmente devido a pequena amostra presente neste estudo. (Tabela 4)

Tabela 4 - Internações hospitalares de acordo com a cor/raça.

\begin{tabular}{llll}
\hline Cor/Raça & Número de internações & Óbitos & Taxa de mortalidade \\
\hline Branca & 1373 & 52 & 3,79 \\
Preta & 333 & 10 & 3,0 \\
Parda & 14.081 & 511 & 3,63 \\
Amarela & 955 & 34 & 3,56 \\
Índigena & 5 & 1 & 20,00 \\
Sem informação & 7492 & 311 & 4,15 \\
\hline
\end{tabular}

Fonte: https://datasus.saude.gov.br

\section{Discussão}

A região Nordeste apresentou uma incidência média anual de 22,22 casos de fratura de fêmur a cada 10000 idosos, valor bem próximo às verificadas no Brasil em sua totalidade que apresentou incidência média geral anual de 22,402 a cada 10000 e as observadas nas cidades de Fortaleza e Joinville, com 21,78 e 21,52 a cada 10000 idosos (Vasconcelos et al., 2020; Silveira et al., 2005; Silva et al., 2019). Uma pesquisa mais antiga realizada na cidade de Marília, São Paulo, mostrou uma incidência levemente superior, variando de 29,48 a 35,83 casos a cada 10000 idosos (Komatsu et al., 1999). Em estudo chinês, a incidência demonstrada foi consideravelmente superior: 278 a cada 100000 casos (Gong et al., 2021). Informações especulativas em estudo apontam como as condições climáticas, devido a uma maior ou menor exposição de luz solar e consequente ação de vitamina $\mathrm{D}$, além das características genéticas e raciais de cada uma dessas populações podem justificar algumas diferenças encontradas nas incidências (Silveira et al., 2005). Contudo, as semelhanças de incidência entre este estudo 
e o de Joinville, por exemplo, deixam essas especulações um tanto contraditórias, já que as populações são consideravelmente diferentes geneticamente, a maioria da população do estudo em Joinville era branca, além de as regiões terem climas diferentes.

Comparando os anos de 2019 e 2020, não houveram grandes diferenças em relação ao número de internações, sendo 12208 e 12031, respectivamente. Diferente do exposto em estudo recente americano, que registrou uma queda de $50 \%$ das internações de pacientes por fratura de quadril quando se compararam as internações antes do Covid-19 e durante a pandemia (Zhong et al., 2021). Segundo o estudo, a queda das internações pode ter ocorrido em virtude da redução das atividades devido à quarentena ou pelo redirecionamento desses pacientes a grandes centros de trauma diferentes dos analisados, uma vez que o número de fraturas registrados no país não reduziu (Zhong et al., 2021). A quarentena, e a consequente diminuição das atividades externas, não influenciaria na diminuição desses resultados, posto que a maioria das fraturas nos idosos ocorre por queda da própria altura, que por sua vez, ocorre majoritariamente em ambiente domiciliar (Maia et al., 2011).

A razão masculino / feminino observada neste estudo de 1:2,2 foi semelhante a de 1:2,01 encontrada no Paraná, a de 1: 2,04 encontrada em Joinville e a de 1:1,7 encontrada em estudo similar no Brasil por completo e em Sobral no Ceará (Oliveira \& Borba, 2017; Silva et al., 2019; Vasconcelos et al., 2020; Rocha \& Ribeiro, 2004). Em pesquisa feita em Salvador na Bahia também foram encontradas proporções semelhantes, 1: 2,55 (Santana et al., 2015). Contudo, essa proporção foi consideravelmente inferior às encontradas em estudos realizados em Fortaleza e em São Paulo (Silveira et al., 2005; Fortes et al., 2008; Daniachi et al., 2015). Recentes estudos, americano e chinês, também mostraram prevalência de fraturas em mulheres em uma proporção aproximadamente duas vezes maior que em homens (Zhong et al., 2021; Gong et al., 2021). O estudo atual discordou do estudo BRAZOS que encontrou uma tendência maior em homens da região Nordeste a apresentar fratura por baixo impacto, contudo, nas outras regiões as mulheres também foram a maioria nas amostras (Pinheiro et al., 2010). A maior prevalência de osteoporose e consequentemente de fraturas em mulheres é algo bem elucidado na literatura, tendo em vista que as mulheres, além de apresentarem um pico de massa óssea menor, na pós-menopausa apresentam uma diminuição acelerada da massa óssea devido ao hipoestrogenismo (o estrógeno tem papel importante no processo de remodelação óssea), além de uma maior susceptibilidade para quedas e uma maior expectativa de vida do que os homens. (Amadei et al., 2006; Fernandes et al., 1995; Radominski et al., 2004; Silveira et al., 2005).

Em relação a cor da pele, foi encontrada uma predominância de pardos (58,09\%), diferindo da proporção encontrada no Paraná e na cidade de Joinville, onde houve predomínio de fraturas na raça branca (64,6\% e 96,7\%, respectivamente) (Oliveira \& Borba, 2017; Silva et al., 2019). A prevalência de brancos também foi vista no estudo mineiro (Rocha et al., 2001). Em Salvador (BA), também na região Nordeste do Brasil, foi verificada uma prevalência de indivíduos pardos (Santana et al., 2015). Nos EUA, houve predomínio de indivíduos brancos, representando por volta de $88 \%$ dos casos, nos dois anos avaliados (Zhong et al., 2021). As porcentagens dos estudos podem ser reflexo da prevalência das raças nas respectivas populações, uma vez que no Sul do País, por exemplo, a população caucasiana é mais prevalente, já no Nordeste há predomínio de pardos. Outro aspecto que pode prejudicar a aplicabilidade dos estudos é a grande miscigenação existente no País, que torna difícil a caracterização dos indivíduos em uma raça apenas. O que se tem elucidado em literatura é que os negros são menos susceptíveis a osteoporose e fraturas por atingirem uma massa óssea maior devido a maior reabsorção renal de cálcio e resistência à ação óssea do paratormônio (PTH) (Hungria Neto et al., 2011; Pinheiro et al., 2010). No entanto, autores encontraram prevalências elevadas para mulheres negras e brancas considerando que a etnia não deve ser critério de exclusão para rastreamento de osteoporose (Faisal-Cury \& Zacchello, 2007).

O número de fraturas aumentou de acordo com o envelhecimento, sendo maior na faixa etária dos 80 anos ou mais que totalizaram 11.677 internações $(48,17 \%)$, semelhante ao estado do Paraná que teve a maior parte dos casos nessa faixa de idade (45,06\%) (Oliveira \& Borba, 2017). Em Fortaleza e Salvador também houve predominância dos indivíduos com 80 anos 
ou mais (Silveira et al., 2005; Santana et al., 2015). Considerando a literatura internacional, no Município de Banes, em Cuba, a faixa etária mais prevalente encontrada em estudo recente foi a de 70 a 79 anos, porém com um grupo estudado de apenas 49 indivíduos com fratura de quadril (Sánchez Delgado et al., 2021). Já nos EUA, os resultados foram mais semelhantes sendo 81 anos a média de idade dos indivíduos estudados (Zhong et al., 2021). O aumento progressivo dos casos de osteoporose e fraturas com a idade ocorre porque a velocidade de perda óssea se torna muito maior na meia idade, contribuindo para o desenvolvimento dessa patologia progressivamente ao longo dos anos (Rodrigues \& Barros, 2016).

A média de permanência na internação neste estudo foi de 8,9 dias, sendo 9,3 em 2019 e 8,6 em 2020. Em Joinville e em São Paulo as médias de permanência foram superiores chegando a 14,1 dias e 13,5 dias, respectivamente (Silva et al., 2019; Daniachi et al., 2015). Em Sobral no estado do Ceará, os pacientes receberam alta mais precocemente com média de 7,2 dias de permanência (Rocha \& Ribeiro, 2004). Em estudo mineiro, a média de dias foi mais próxima: 10 dias (Rocha et al., 2001). Nos EUA, durante a pandemia os pacientes receberam alta hospitalar um dia antes comparado ao ano anterior, visando tirar precocemente esses indivíduos do ambiente hospitalar devido ao risco de infecção pelo Covid-19, semelhante ao que ocorreu neste estudo, apesar de a média de dias de permanência dos estadunidenses ser consideravelmente menor (5 e 4 dias) (Zhong et al., 2021). De acordo com pesquisa é possível inferir que um dos fatores que mais influenciam no tempo de permanência é o adiamento do procedimento cirúrgico que acarreta em maior dificuldade de estabilização do paciente após o procedimento e um consequente maior tempo de internação (Fonseca et al., 2000). Por outro lado, tempos de permanência muito curtos (1 a 7 dias) revelam que o paciente pode ter ido para casa sem a garantia de uma assistência adequada, tendo em vista que seria necessário encaminhar o paciente para reabilitação e tratamento clínico da osteoporose, com objetivo de evitar a ocorrência de novas fraturas (Bortolon et al., 2011).

De todos os 24239 pacientes idosos internados por fratura de fêmur, 919 vieram a óbito, resultando em uma taxa de mortalidade durante a internação de 3,79\%. O valor encontrado se mostrou levemente inferior ao encontrado no Paraná (5,9\%), em Joinville (7,5\%), em São Paulo (7,1\%) e no Brasil no geral (4,99\%) (Oliveira \& Borba, 2017; Silva et al., 2019; Daniachi et al., 2015; Vasconcelos et al., 2020). Em Sobral no Ceará a taxa de mortalidade foi consideravelmente inferior, 2,5\% dos casos (Rocha \& Ribeiro, 2004). Mesmo a maioria dos casos de fratura sendo entre mulheres, a taxa de mortalidade foi superior no sexo masculino (3,97\%). Concordou com o estudo no Paraná, que também encontrou mortalidade maior em homens apesar de um pouco superior $(6,02 \%)$ (Oliveira \& Borba, 2017). Porém, em estudos anteriores realizados no Brasil e na cidade de São Paulo a mortalidade foi superior nas mulheres (Bortolon et al., 2011; Fortes et al., 2008). Segundo estudo, a existência de outras doenças crônicas nos pacientes idosos no momento da fratura é um fator prognóstico importante, sendo as afecções cardíacas e pulmonares, doenças renais, diabetes mellitus e acidente vascular cerebral (AVC) as de maior influência na mortalidade (Sakaki et al., 2004).

Em relação a cor da pele, a taxa de mortalidade neste estudo foi maior em indígenas (20\%), provavelmente devido a pequena amostra encontrada de apenas 5 internações e um óbito. Outro empecilho, foram as internações sem informações sobre a cor, que totalizaram 7492 e 311 óbitos. Excluindo essas internações sem informações, a segunda maior taxa de mortalidade encontrada foi em brancos $(3,79 \%)$. Os dados do Paraná discordaram trazendo prevalência maior entre negros e asiáticos (7,59 e 7,27\%) (Oliveira \& Borba, 2017).

Os gastos totais com internações por fratura de fêmur foram $\mathrm{R} \$ 62.713 .218,03$ nos dois anos estudados, resultando em uma média de $\mathrm{R} \$ 2587,29$ por idoso internado. No Paraná o custo médio da internação foi bem próximo chegando a $\mathrm{R} \$$ 2.618,34 (Oliveira \& Borba, 2017). Esses dados elucidam como as despesas geradas pelas fraturas de fêmur são onerosas para o sistema de saúde. 


\section{Conclusão}

Neste estudo a incidência encontrada foi de 22,22 casos a cada 10000 idosos, advinda de um valor absoluto de 24239 internações. As mulheres protagonizaram as internações com 2,2 vezes mais casos em relação aos homens. A diferença do número de internações entre os anos estudados foi pequena, apenas 177 casos. Houve predomínio de indivíduos pardos, que representaram 58,09\% dos casos. O número de internações aumentou progressivamente com a idade, sendo mais comum na faixa etária de 80 anos ou mais (48,17\% dos casos). Os pacientes ficaram internados por uma média de 9,3 dias em 2019 e 8,6 dias em 2020, um decréscimo de cerca de 1 dia. A taxa de mortalidade durante a internação foi de 3,79\%, e foi superior no sexo masculino $(3,97 \%)$, em indígenas e brancos. Os gastos do SUS devido a internações por fratura de fêmur em idosos da região Nordeste do Brasil nesses dois anos foi de R\$62.713.218,03, se mostrando bastante onerosos.

Sugere-se que futuramente sejam realizadas novas pesquisas sobre a epidemiologia das fraturas do fêmur em idosos objetivando definir melhor uma população a ser triada para aplicação de políticas públicas de rastreio e prevenção de osteoporose e suas consequentes fraturas.

\section{Referências}

Amadei, S. U., Silveira, V. Á. S., Pereira, A. C., Carvalho, Y. R., \& Rocha, R. F. da. (2006). A influência da deficiência estrogênica no processo de remodelação e reparação óssea. Jornal Brasileiro de Patologia e Medicina Laboratorial, 42(1). https://doi.org/10.1590/S1676-24442006000100003

Barrionuevo, P., Kapoor, E., Asi, N., Alahdab, F., Mohammed, K., Benkhadra, K., Almasri, J., Farah, W., Sarigianni, M., Muthusamy, K., Al Nofal, A., Haydour, Q., Wang, Z., \& Murad, M. H. (2019). Efficacy of Pharmacological Therapies for the Prevention of Fractures in Postmenopausal Women: A Network Meta-Analysis. The Journal of Clinical Endocrinology \& Metabolism, 104(5), 1623-1630. https://doi.org/10.1210/jc.2019-00192

Bortolon, P. C., Andrade, C. L. T. de, \& Andrade, C. A. F. de. (2011). O perfil das internações do SUS para fratura osteoporótica de fêmur em idosos no Brasil: Uma descrição do triênio 2006-2008. Cadernos de Saúde Pública, 27, 733-742. https://doi.org/10.1590/S0102-311X2011000400012

Daniachi, D., Santos Netto, A. dos, Ono, N. K., Guimarães, R. P., Polesello, G. C., \& Honda, E. K. (2015). Epidemiologia das fraturas do terço proximal do fêmur em pacientes idosos 论. Revista Brasileira de Ortopedia, 50, 371-377. https://doi.org/10.1016/j.rboe.2015.06.007

DATASUS - Ministério da Saúde. ([s.d.]). Recuperado 7 de outubro de 2021, de https://datasus.saude.gov.br/

Faisal-Cury, A., \& Zacchello, K. P. (2007). Osteoporose: Prevalência e fatores de risco em mulheres de clínica privada maiores de 49 anos de idade. Acta Ortopédica Brasileira, 15, 146-150. https://doi.org/10.1590/S1413-78522007000300005

Fernandes, C. E., Wehba, S., \& Melo, N. R. de. (1995). Fisiopatologia da osteoporose pós-menopausa. Reprod. clim, 10(4), 153-159.

Fonseca, F. F., Má, L. S., Pereira, X., \& Arantes, P. M. M. (2012). Clinical outcomes related factors in elderly with hip fracture assisted in a university hospital. Geriatrics, Gerontology and Aging, 6(4), 352-360.

Fortes, É. M., Raffaelli, M. P., Bracco, O. L., Takata, E. T. T., Reis, F. B., Santili, C., \& Lazaretti-Castro, M. (2008). Elevada morbimortalidade e reduzida taxa de diagnóstico de osteoporose em idosos com fratura de fêmur proximal na cidade de São Paulo. Arquivos Brasileiros de Endocrinologia \& Metabologia, 52, 1106-1114. https://doi.org/10.1590/S0004-27302008000700006

Gong, X. F., Li, X. P., Zhang, L. X., Center, J. R., Bliuc, D., Shi, Y., Wang, H. B., He, L., \& Wu, X. B. (2021). Current status and distribution of hip fractures among older adults in China. Osteoporosis International, 32(9), 1785-1793. https://doi.org/10.1007/s00198-021-05849-y

Hungria Neto, J. S., Dias, C. R., \& Almeida, J. D. B. de. (2011). Características epidemiológicas e causas da fratura do terço proximal do fêmur em idosos. Revista Brasileira de Ortopedia, 46, 660-667. https://doi.org/10.1590/S0102-36162011000600007

IBGE. ([s.d.]). Recuperado 7 de outubro de 2021, de https://www.ibge.gov.br/apps/snig/v1/?loc=0,2\&cat=1,2,-2,8,128\&ind=4711

Kanis, J. A., Cooper, C., Rizzoli, R., \& Reginster, J.-Y. (2019). European guidance for the diagnosis and management of osteoporosis in postmenopausal women. Osteoporosis International, 30(1), 3-44. https://doi.org/10.1007/s00198-018-4704-5

Komatsu, R. S., Simões, M. F. J., Ramos, L. R., \& Szejnfeld, V. L. (1999). Incidência de fraturas do fêmur proximal em Marília, São Paulo, Brazil, 1994 e 1995. Rev. bras. reumatol, 325-331.

Lima-Costa, M. F., \& Barreto, S. M. (2003). Tipos de estudos epidemiológicos: Conceitos básicos e aplicações na área do envelhecimento. Epidemiologia e Serviços de Saúde, 12(4). https://doi.org/10.5123/S1679-49742003000400003

Maia, B. C., Viana, P. S., Arantes, P. M. M., \& Alencar, M. A. (2011). Consequências das quedas em idosos vivendo na comunidade. Revista Brasileira de Geriatria e Gerontologia, 14(2), 381-393. https://doi.org/10.1590/S1809-98232011000200017

Miranda, G. M. D., Mendes, A. da C. G., \& Silva, A. L. A. da. (2016). O envelhecimento populacional brasileiro: Desafios e consequências sociais atuais e futuras. Revista Brasileira de Geriatria e Gerontologia, 19, 507-519. https://doi.org/10.1590/1809-98232016019.150140 
Oliveira, C. C., \& Borba, V. Z. C. (2017). Epidemiology Of Femur Fractures In The Elderly And Cost To The State Of Paraná, Brazil. Acta Ortopédica Brasileira, 25, 155-158. https://doi.org/10.1590/1413-785220172504168827

Pinheiro, M. M., Ciconelli, R. M., Jacques, N. de O., Genaro, P. S., Martini, L. A., \& Ferraz, M. B. (2010). O impacto da osteoporose no Brasil: Dados regionais das fraturas em homens e mulheres adultos - The Brazilian Osteoporosis Study (BRAZOS). Revista Brasileira de Reumatologia, 50, 113-120. https://doi.org/10.1590/S0482-50042010000200002

Radominski, S. C., Pinto-Neto, A. M., Marinho, R. M., Costa-Paiva, L. H. S., Pereira, F. A., Urbanetz, A. A., Ferrari, A. E. M., \& Baracat, E. C. (2004). Osteoporose em mulheres na pós-menopausa. Revista Brasileira de Reumatologia, 44, 426-434.

Rocha, F. A. C. da, \& Ribeiro, A. R. (2004). Baixa incidência de fraturas do quadril associadas à osteoporose, em Sobral-CE. Revista Brasileira de Reumatologia, 44, 255-258.

Rocha, M. A., Carvalho, W. S., Zanqueta, C., \& Lemos, S. C. (2001). Estudo epidemiológico retrospectivo das fraturas do fêmur proximal tratados no Hospital Escola da Faculdade de Medicina do Triângulo Mineiro. 36, 6.

Rodrigues, I. G., \& Barros, M. B. de A. (2016). Osteoporose autorreferida em população idosa: Pesquisa de base populacional no município de Campinas, São Paulo. Revista Brasileira de Epidemiologia, 19, 294-306. https://doi.org/10.1590/1980-5497201600020007

Sakaki, M. H., Oliveira, A. R., Coelho, F. F., Leme, L. E. G., Suzuki, I., \& Amatuzzi, M. M. (2004). Estudo da mortalidade na fratura do fêmur proximal em idosos. Acta Ortopédica Brasileira, 12, 242-249. https://doi.org/10.1590/S1413-78522004000400008

Sánchez Delgado, J. A., Pérez Almoza, G., Sánchez Lara, N. E., Sánchez Delgado, J. A., Pérez Almoza, G., \& Sánchez Lara, N. E. (2021). Comportamiento epidemiológico de la fractura de cadera. Revista Cubana de Ortopedia y Traumatología, 35(1). http://scielo.sld.cu/scielo.php?script=sci_abstract\&pid=S0864$215 X 2021000100008 \& \operatorname{lng}=\mathrm{es} \& n \mathrm{rm}=\mathrm{iso} \& \operatorname{lng}=\mathrm{es}$

Santana, D. F., Reis, H. F. C. dos, Ezequiel, D. J. S., \& Ferraz, D. D. (2015). Perfil funcional, sociodemográfico e epidemiológico de idosos hospitalizados por fratura proximal de fêmur. Revista Kairós-Gerontologia, 18(1), 217-234. https://doi.org/10.23925/2176-901X.2015v18i1p217-234

Silva, D. M. W., Lazaretti-Castro, M., Freitas Zerbini, C. A. de, Szejnfeld, V. L., Eis, S. R., \& Borba, V. Z. C. (2019). Incidence and excess mortality of hip fractures in a predominantly Caucasian population in the South of Brazil. Archives of Osteoporosis, 14(1), 47. https://doi.org/10.1007/s11657-019-0597-y

Silveira, V. A. L., Medeiros, M. M. das C., Coelho-Filho, J. M., Mota, R. S., Noleto, J. C. S., Costa, F. S. da, Pontes, F. J. O. de, Sobral, J. B., Aguiar, R. F., Leal, Â. C., \& Clemente, C. M. (2005). Incidência de fratura do quadril em área urbana do Nordeste brasileiro. Cadernos de Saúde Pública, $21,907-912$. https://doi.org/10.1590/S0102-311X2005000300025

Vasconcelos, P. A. B. de, Rocha, A. de J., Fonseca, R. J. de S., Teixeira, T. R. G., Mattos, E. de S. R., \& Guedes, A. (2020). Femoral fractures in the elderly in Brasil-Incidence, lethality, and costs (2008-2018). Revista Da Associação Médica Brasileira, 66, 1702-1706. https://doi.org/10.1590/1806-9282.66.12.1702

Zhong, H., Poeran, J., Liu, J., Wilson, L. A., \& Memtsoudis, S. G. (2021). Hip fracture characteristics and outcomes during COVID-19: A large retrospective national database review. British Journal of Anaesthesia, 127(1), 15-22. https://doi.org/10.1016/j.bja.2021.04.003 